\title{
The Economics of Career Concerns, Part I: Comparing Information Structures
}

\author{
MATHIAS DEWATRIPONT \\ ECARE, Universite Libre de Bruxelles, CEPR and CORE \\ IAN JEWITT \\ University of Bristol, CEPR and CMPO \\ and \\ JEAN TIROLE \\ IDEI, GREMAQ, CERAS, MIT
}

First version received March 1998; final version accepted October 1998 (Eds.)

\begin{abstract}
Many incentives in organizations arise not through explicit formal incentive contracts but rather implicitly through career concerns. This paper models career concerns through agents trying to manipulate the market assessment of their future productivity. The information flow from current actions to market assessment is therefore crucial in determining the nature of these incentives. Improved information may either increase or reduce incentives. The impact of information provides a major distinction between the explicit and implicit incentives model. The paper derives general results on comparisons of information structures which serve as counterparts to the standard results on information structures in the principal-agent model: sufficient statistic, impact of a Blackwell garbling, comparison of inclusive information structures.
\end{abstract}

\section{INTRODUCTION}

The past 25 years have witnessed the development of incentives theory. Most attention has been devoted to formal or explicit incentives, such as piece rate wages, bonuses and stock options. A broad set of insights has been derived for the paradigm of explicit incentive schemes under moral hazard ${ }^{1}$ and adverse selection. ${ }^{2}$ Equally important in reality are implicit incentives, in the form of career concerns, inside or outside the organization. These play a key role in all organizations, private and public, but are particularly strong in the government sector, where formal incentives schemes are often crude and constrained. In this sector, elections, promotions, and future employment in the private sector are major motivations to expend effort in the current job.

In a seminal paper, Holmstrom (1982b) provided a tractable model of career concerns, whose simplest version goes as follows. There are two periods, today and tomorrow. Today's performance $(y)$ by a manager, is the sum of her talent $(\theta)$, and of current effort (a). The distribution of talent has full support, and talent is unknown to everybody (so

1. See for example Mirrlees (1999), Shavell (1979), Holmstrom (1979, 1982a), Grossman-Hart (1983), Jewitt (1988, 1997), Holmstrom-Milgrom (1991), Kim (1995).

2. See e.g. Mirrlees (1971) and Guesnerie-Laffont (1984). 
there is symmetric information at date 1). Effort is observed only by the manager, and costs her $\psi(a)$. Performance is observable by everyone but not describable ex ante in a formal compensation contract. The manager is thus paid a fixed wage $t_{1}$ today, and so the manager exerts effort solely to influence her wage tomorrow. For simplicity, assume this wage $t_{2}(y)$ is the market's assessment of her productivity, $E(\theta \mid y)$. If $\delta$ is the discount factor between the two periods, the manager maximizes

$$
t_{1}-\psi(a)+\delta t_{2}(y)
$$

In a pure strategy equilibrium with equilibrium effort $a^{*}, t_{2}(y)=y-a^{*}=\theta+a-a^{*}$ and so $\psi^{\prime}\left(a^{*}\right)=\delta$. Holmstrom $(1982 b)$ generalized this model in a multiperiod setting to the case of a normally distributed talent and an additive normally distributed noise to performance. While Holmstrom's paradigm has generated a number of applications, it has received much less attention than that of formal incentives. This paper generalizes the career concerns model, in order to evaluate the robustness of the insights derived from the Holmstrom model and to compare the career concerns paradigm with the formal incentives one and provide a basis for further research.

The core of this paper, Sections 3 through 6, compares information structures. Two results on information systems are prominent in the explicit incentives literature. The sufficient statistic theorem of Holmstrom (1979) and Shavell (1979) states that an observable $z$ is redundant in the construction of an agent's incentive scheme given the availability of another observable $y$ if and only if $y$ is a sufficient statistic for $(y, z)$ when estimating effort. Second, a Blackwell garbling of the information system raises agency costs (e.g. Grossman-Hart (1983)). More recently, Kim (1995) shows that, provided the first-order approach is valid, agency costs increase (information deteriorates) when the distribution of the likelihood ratio incurs a mean-preserving spread. We may wonder whether analogous results hold in the implicit incentives model.

Our propositions on the comparison of information systems have two main benefits. First, they identify the general determinants of implicit incentives. Second, they allow comparisons of the impact of alternative information structures on career concerns in specific applied models for which, as is usually the case, closed form solutions are unavailable. Section 4 introduces a key ingredient for the comparison of information structures: the likelihood ratio martịngale property. Section 4 derives a sufficient statistic result, paralleling that of Holmstrom (1979) for the case of explicit incentives. Section 5 obtains general conditions under which Blackwell garblings of information increase or decrease implicit incentives. Section 6 shows that weaker comparisons of information structures can do if we make stronger assumptions on the distributions of some random variables, e.g. Fisher information for exponential families. Lastly, Section 7 summarizes our analysis and concludes.

\section{THE MODEL}

\subsection{Description}

There are two parties, called the "agent" and the "market" (or the "organization", or the "principal"). The agent chooses an unobservable vector of "actions" or "efforts" $a=$ $\left(a_{1}, \ldots, a_{n}\right) \in \mathbb{R}^{n}$ and incurs private cost $c(a)$. The market then observes a vector of observables or performance variables $y=\left(y_{1}, \ldots, y_{m}\right) \in \mathbb{R}^{m}$ and takes actions that result in benefit or reward $t$ for the agent, whose utility is then

$$
t-c(a) \text {. }
$$


The reward $t$ reflects the market's expectation of an unknown parameter $\theta$ conditional on the observables $y$. As in the standard career concerns model, $\theta$ will be referred to as the "agent's talent" and in the entire paper is taken to be a scalar. Quite generally, let $f(\theta, y \mid a)$ denote the joint density of talent and observables given effort vector $a$. Let

$$
\hat{f}(y \mid a)=\int f(\theta, y \mid a) d \theta,
$$

denote the marginal density of the observables. The agent's reward for performance variables $y$ and equilibrium actions $a^{*}$ is thus

$$
t=E\left(\theta \mid y, a^{*}\right)=\int \theta \frac{f\left(\theta, y \mid a^{*}\right)}{f\left(y \mid a^{*}\right)} d \theta .
$$

Let $c_{a}$ and $f_{a}$ denote the gradients with respect to efforts of the cost function and of the marginal distribution ("subscripts $a$ " will be replaced by "primes" when $n=1$ ).

Remark. In many applications of the model (including Holmstrom's original career concerns model), the density $f$ is separable

$$
f(\theta, y \mid a)=g(\theta) h(y \mid \theta, a) .
$$

That is, the agent's talent $\theta$ is drawn from some distribution $g(\cdot)$ independent of $a$ and performance $y$ is a stochastic function of talent and effort. Our more general formulation allows for situations in which effort affects the reward even if it is observed. For instance, in a career concerns model with learning by doing, $\theta$ stands for the agent's "second-period talent", which results from her intrinsic talent and the "first-period effort".

Example 2.1. The single-task additive-normal career concerns model. The two-period version of Holmstrom's model has $n=m=1$ and an additive-normal structure. There are two dates, $\tau=1,2$. The agent's effort $a_{\tau} \geqq 0$ generates observable but noncontractible profit $y_{\tau}$ for her date- $\tau$ employer, where

$$
\begin{gathered}
y_{\tau}=\theta+a_{\tau}+\varepsilon_{\tau}, \\
\theta \sim \mathscr{N}\left(\theta, \sigma_{\theta}^{2}\right) \text { and } \varepsilon_{\tau} \sim \mathscr{N}\left(0, \sigma_{\varepsilon}^{2}\right),
\end{gathered}
$$

and $\theta$ and the $\varepsilon_{\tau}$ s are all independently distributed.

In the "first period" of the model, the agent receives a fixed wage $t_{1}$. She then exerts effort $a_{1} \geqq 0$ at private cost $\psi\left(a_{1}\right)$. "Period two" being the last period, she no longer has career concerns and she exerts $a_{2}=0$. Her second-period wage is determined in a competitive labour market and is equal to her expected marginal productivity conditional on her first period performance $y_{1}$ and equilibrium effort $a_{1}^{*}$

$$
t_{2}=E\left(\theta \mid y_{1}, a_{1}^{*}\right) .
$$

The discount factor between the two periods is $\delta$ and so the agent's utility is

$$
t_{1}+\delta E\left(t_{2}\right)-\psi\left(a_{1}\right) .
$$

The Holmstrom model fits the general model with $t \equiv t_{2}, a \equiv a_{1}, y \equiv y_{1}, c(a)=\psi(a) / \delta$. The joint distribution of $\theta$ and $y$ given $a$, which is bivariate normal, satisfies

$$
f(\theta, y \mid a) \propto \exp \left[-(\theta-\theta)^{2} / 2 \sigma_{\theta}^{2}\right] \exp \left[-(y-\theta-a)^{2} / 2 \sigma_{\varepsilon}^{2}\right],
$$

where " $\propto$ " stands for "proportional to". 
Remark. The model is thus a "two-period" model (effort in period 1, reward in period 2). Its extension to more than two periods is, except in the additive normal case, a complex matter that is left for future research. In a multi-period context, the agent acquires private information about the marginal productivity of her effort and the impact of effort on future wages - except when effort affects performance additively and distributions are normal - and so the agent's dynamic optimization programme must account for the impact of alternative choices of effort on her learning of information about productivity.

\subsection{A general formula}

Suppose that the market anticipates equilibrium effort vector $a^{*}$. The agent chooses $a$ so as to maximize her expected utility

$$
\max E\left[E\left(\theta \mid y, a^{*}\right)\right]-c(a)
$$

where the first expectation is with respect to performance and the second with respect to talent. Assuming an interior solution, ${ }^{3}$ the first-order condition for an equilibrium is

$$
\left.\frac{d}{d a}\left(\int\left(\int \theta \frac{f\left(\theta, y \mid a^{*}\right)}{f\left(y \mid a^{*}\right)} d \theta\right) \hat{f}(y \mid a) d y\right)\right|_{a=a^{*}}=c_{a}\left(a^{*}\right),
$$

or

$$
\iint \theta f\left(\theta, y \mid a^{*}\right) \frac{\hat{f_{a}}\left(y \mid a^{*}\right)}{f\left(y \mid a^{*}\right)} d y d \theta=c_{a}\left(a^{*}\right) .
$$

Using the fact that the (multidimensional) likelihood ratio has zero mean $\left(E\left(f_{a} / f\right)=0\right)$, we can rewrite the equilibrium condition as

$$
\operatorname{cov}\left(\theta, \frac{f_{a}}{f}\right)=c_{a}\left(a^{*}\right)
$$

where "cov" denotes the covariance of two random variables. ${ }^{4}$ The vector $\operatorname{cov}\left(\theta, f_{a} / f\right)$ describes the agent's marginal incentives.

So, our first result is:

Proposition 2.1 (Equilibrium condition). In an equilibrium of the career concerns model, the gradient of the cost function is equal to the covariance of talent and the likelihood

3. If we impose a lower bound on the effort vector $a \geqq \underline{a}$, as we will do in some of our examples, Proposition 1 must be written with complementary slackness:

$$
(a-\underline{a}) \cdot\left[\operatorname{cov}\left(\theta, \frac{\hat{f}}{f}\right)-c_{a}\left(a^{*}\right)\right]=0 .
$$

4. It is interesting to compare condition (2.2) for an implicit incentive scheme with the standard formula for explicit incentive schemes (Mirrlees (1999), Holmstrom (1979), Shavell (1979), Jewitt (1988)). Suppose the agent receives an explicit transfer $t(y)$ contingent on performance $y$ and has utility $u(t)$ from income $t$. Then the first-order condition for the agent is

$$
\operatorname{cov}\left(u(t(y)), \frac{\hat{f_{a}}}{f}\right)=c_{a}\left(a^{*}\right) .
$$

This comparison suggests why (a version of) the sufficient statistic result for explicit incentives carries over to implicit ones (see Proposition 5.1). 
ratio

$$
\operatorname{cov}\left(\theta, \frac{\hat{f_{a}}}{f}\right)=c_{a}\left(a^{*}\right)
$$

Application to the single-task additive-normal model. With $n=m=1$ and $y=\theta+a \neq \varepsilon$ with $\theta$ and $\varepsilon$ independent random variables, $y$ is normally distributed with mean $\theta+a$ and variance $\sigma_{\theta}^{2}+\sigma_{\varepsilon}^{2}$. Consequently, $f(y \mid a)$ is proportional to

$$
\exp \left[-\frac{(y-\bar{\theta}-a)^{2}}{2\left(\sigma_{\theta}^{2}+\sigma_{\varepsilon}^{2}\right)}\right],
$$

and

$$
\frac{\hat{f_{a}}}{f}=\frac{(\theta-\bar{\theta})+\varepsilon}{\sigma_{\theta}^{2}+\sigma_{\varepsilon}^{2}} .
$$

We thus obtain Holmstrom's standard result

$$
\operatorname{cov}\left(\theta, \frac{\hat{f_{a}}}{f}\right)=\frac{\sigma_{\theta}^{2}}{\sigma_{\theta}^{2}+\sigma_{\varepsilon}^{2}} .
$$

And so, from (2.2),

$$
\psi^{\prime}\left(a^{*}\right)=\delta \frac{\sigma_{\theta}^{2}}{\sigma_{\theta}^{2}+\sigma_{\varepsilon}^{2}} .
$$

Making the natural assumption that the cost function is strictly convex, we see that the equilibrium is unique and that the effort $a^{*}$ increases when the performance becomes more informative in the sense that the variance of the measurement error $\varepsilon$ decreases. Uniqueness of equilibrium does however rely on the fact that effort and talent enter additively in the determination of output: as stressed in our companion paper (Dewatripont et al. (1999)), complementarity between effort and talent can lead to multiple equilibria. In this paper however, we shall restrict attention to comparative statistics around a given equilibrium.

Remark. The Schwartz inequality

$$
\begin{aligned}
\operatorname{cov}\left(\theta, \frac{\hat{f_{a}}\left(y \mid a^{*}\right)}{f\left(y \mid a^{*}\right)}\right) & =E\left[E\left[\theta \mid y, a^{*}\right] \cdot \frac{\hat{f_{a}}\left(y \mid a^{*}\right)}{f\left(y \mid a^{*}\right)}\right] \\
& \leqq \sqrt{\operatorname{var}(t) \cdot E\left(\frac{\hat{f_{a}}}{f}\right)^{2}}
\end{aligned}
$$

allows us to obtain an upper bound on incentives as a function of wage variability: The marginal incentive is bounded by the product of the standard deviation of (future) wages and the square root of the Fisher information. This bound is increasing in information since the more information, the larger both the Fisher information and the variance of future wages (unless the upper bound is reached, this of course does not imply that the marginal incentive is increasing in Fisher information). Equality holds if and only if there are scalars $A$ and $B$ and $C$ ( $A$ and $B$ having opposite signs) such that $A E\left[\theta \mid y, a^{*}\right]+$ $B\left(f_{a}\left(y \mid a^{*}\right)\right) / f\left(y \mid a^{*}\right)=C$. We will identify cases in which this bound is tight below. 


\section{PRELIMINARIES: INFORMATION AND LIKELIHOOD RATIO MARTINGALES}

The rest of this paper is devoted to a study of the comparison of alternative information structures in terms of marginal incentives. Equation (2.2) suggests that one of the key factors in the comparison of information structures is the comparison of their likelihood ratios. This section reminds the reader of some background material on likelihood ratio martingales that will be used in later sections. In particular, it links the Blackwell, Lehman and Fisher comparisons of information structures (from strongest to weakest) to the properties of likelihood ratios. The following sections relate the three comparisons to marginal incentives.

Blackwell information. The agent's effort $a$ determines the distribution of observable statistics. These statistics constitute, in Blackwell's terminology, experiments which provide information about $a$. Suppose for instance that the statistic $y$ with marginal density $f(y \mid a)$ is more informative than the statistic $z$ with marginal density $g(z \mid a)$. Then, by Blackwell's theorem, there exists a joint distribution for $(y, z)$ with marginal distributions $f$ and $g$ such that $y$ is sufficient for $(y, z)$. Thus, let us assume that there exists a conditional density $k(z \mid y)$ such that

$$
\hat{g}(z \mid a)=\int k(z \mid y) \hat{f}(y \mid a) d y .
$$

(3.1) implies a martingale property for the (multivariate) likelihood ratios

$$
\frac{\hat{g_{a}}(z \mid a)}{\hat{g}(z \mid a)}=E\left[\frac{\hat{f_{k}}(y \mid a)}{f(y \mid a)} \mid z, a\right],
$$

where the expectation is taken with respect to the joint density $k(z \mid y) f(y \mid a)$.

Lehman information. Requiring that the two statistics be ordered by Blackwell information guarantees the martingale condition (3.2), but is stronger than needed. For instance, consider scalars $y=a+\varepsilon$, and $z=a+\eta$. The statistics $y$ and $z$ constitute what Lehman (1988) calls location experiments, and it is known that $y$ is Blackwell more informative than $z$ if and only if $\eta$ has the same distribution as $\varepsilon+\zeta$ for some other random variable $\zeta$ with $\varepsilon$ and $\zeta$ independently distributed. This implies for instance that only a normally distributed $y$ can be Blackwell more informative than a normally distributed $z$ however concentrated or spread out the distributions are. However, condition (3.2) can be shown to hold whenever $y$ and $z$ have monotone likelihood ratio $^{5}$ and $y$ is less dispersed than $z$ (see Proposition 3.1 below). The martingale condition is therefore a substantially weaker requirement than Blackwell information-it orders more experiments.

In many cases it will be natural to think in terms of effort increasing some statistic, for instance sales, in some stochastic sense. We will often assume therefore that effort $a$ increases $z$ in likelihood ratio; this is a common assumption stronger than first-order stochastic dominance.

Definition 3.1 (MLRP). Effort increases z if the marginal distribution $\hat{g}(z \mid a)$ has an increasing likelihood ratio $\left(g_{a} / g\right.$ is increasing in $\left.z\right)$.

5. The implication in Proposition 3.1 goes one way if only $z$ has MLRP. 
Proposition 3.1 (Lehman (1988), Jewitt (1997)). Suppose that the marginal distributions $f(y \mid a)$ and $g(z \mid a)$ satisfy MLRP. Then, the martingale condition,

$$
\frac{\hat{g}_{a}(z \mid a)}{\hat{g}(z \mid a)}=E\left(\frac{\hat{f}_{a}(y \mid a)}{f(y \mid a)} \mid z\right) \text { for all } a \text { and } z,
$$

is equivalent to:

(a) Kim (1995)'s comparison of local informations: The distribution of $\hat{f}_{a} / \hat{f}$ is a meanpreserving spread of the distribution of $g_{a} / g$.

If we assume further a scalar statistic and effort, it is equivalent to

(b) Lehman (1988)'s criterion (comparison of Lorenz curves): For all $a_{1}<a_{2}$,

$$
\hat{F}\left(\hat{F}^{-1}\left(z \mid a_{2}\right) \mid a_{1}\right) \geqq \hat{G}\left(\hat{G}^{-1}\left(z \mid a_{2}\right) \mid a_{1}\right) \text { for all } z \text {. }
$$

Condition (b) can be written in a number of alternative ways (Lehman (1988), Jewitt (1997)) including the following transformation criterion: There exists a function $\mathscr{L}(\cdot, \cdot)$ increasing in its two arguments such that

$$
y \stackrel{d}{=} \mathscr{L}(z, a) .
$$

The martingale condition (3.2) is useful in establishing the linear relationships between likelihood ratios and conditional expectations which determine the cases of equality in the Schwartz inequality (2.4). To see this, note that if we have, say $z=\varphi(\theta+a, \varepsilon)$ for some function $\varphi$ and some noise variable $\varepsilon$, then observing the statistic $y=\theta+a$ is clearly more informative about $a$ than observing $z$. Hence the martingale equation holds and with $\theta$ normally distributed, we have

$$
\frac{\hat{g_{a}}}{\hat{g}}=E\left[\frac{\hat{f_{a}}}{f} \mid z\right]=E\left[\frac{y-\bar{y}}{\sigma_{y}^{2}} \mid z\right],
$$

where $f$ is the density of $y$ and the second equality follows from the familiar properties of normal distributions (or more generally linear exponential families). Hence,

$$
\sigma_{\theta}^{2} \frac{\hat{g}_{a}}{g}=E(\theta \mid z)-\bar{\theta}
$$

which establishes the linear relationship we sought.

Fisher information. If one is willing to restrict distributions to belong to specific families (such as normal or exponential distributions), still weaker comparisons of information structures may suffice for comparing marginal incentives. A standard measure of information, due to Fisher, is the expectation of the square of the likelihood ratio; namely, $y$ is more informative than $z$ in the sense of Fisher if

$$
E\left[\left(\frac{\hat{g_{a}}(z \mid a)}{\hat{g}(z \mid a)}\right)^{2}\right] \leqq E\left[\left(\frac{\hat{f_{A}}(y \mid a)}{f(y \mid a)}\right)^{2}\right] .
$$

From Jensen's inequality, the martingale condition (3.2) implies that $y$ is Fisher more informative than $z$, although the converse need not hold. From (2.2), it is clear that Fisher information plays a central role when the posterior expectation of talent is linear in the likelihood ratio, a fact that we will later exploit. 


\section{SUFFICIENT STATISTICS}

We are concerned with the effect of information on the supply of effort induced by implicit incentives. Holmstrom (1979) showed that in the explicit incentive (moral-hazard principal-agent) problem both effort and the reward of the agent depend only on a sufficient statistic. This is a classic result which can be understood as follows. If $y$ is sufficient for $(y, z)$ in estimating effort $a$, then the conditional distribution of $z$ on $y$ is independent of $a$. Hence, once $y$ is observed, effort cannot influence the distribution of $z$ and there is no benefit to the principal in making rewards depend on what the agent cannot influence. In the implicit incentive problem, the relationship between what determines effort, what determines (future) wages, and statistical sufficiency is more complicated. There are two unobservables, $a$ and $\theta$, compared to only one in the explicit incentive framework, $a$. Sufficiency results correspondingly arise for two reasons. The first is as in the explicit incentive model. If conditional on $y$, the agent cannot influence the distribution of $z$, then whether or not the market has $z$ in addition to $y$ to condition its inferences about future productivity will be irrelevant to the effort choices of the agent. The second arises through the market's forming expectations about talent. If conditional on $y$, the market's estimate of the agent's talent is independent of $z$, then the agent will have no incentive to manipulate the distribution of $z$ through effort choices even if she is able to do so.

Definition 4.1 (Effort sufficiency). Let $(y, z, \theta)$ have density $h(y, z, \theta \mid a)$. The statistic $y$ is a sufficient statistic for $(y, z)$ when estimating $a$, if the marginal density $h(y, z \mid a)$ can be factored as follows

$$
h(y, z \mid a)=m(y \mid a) l(y, z)
$$

that is, if

$$
h(y, z, \theta \mid a)=k(\theta \mid y, z, a) m(y \mid a) l(y, z)
$$

for some conditional densities $k(\theta \mid y, z, a)$ and $m(y \mid a)$, and density $l(y, z)$.

This is the classical definition of sufficiençy and, once $\theta$ has been marginalized out of the density, is the same as used by Holmstrom (1979).

Proposition 4.1. Suppose that $y$ is a sufficient statistic for $(y, z)$ when estimating $a$; then making $z$ observable when $y$ already is does not alter marginal incentives.

Proof. Let $h(y, z, \theta \mid a)$ denote the joint density of $(y, z, \theta)$ given $a$, and let $f(y, \theta \mid a)$ denote the marginal density of $(y, \theta)$. We have

$$
h(y, z \mid a)=m(y \mid a) l(y, z)
$$

implying

$$
\frac{\hat{h_{a}}}{h}=\frac{m_{a}}{m}=\frac{\hat{f_{a}}}{f}
$$

and so

$$
\operatorname{cov}\left(\theta, \frac{\hat{h_{a}}}{h}\right)=E\left(\theta \frac{\hat{h_{a}}}{h}\right)=E\left(\theta \frac{\hat{f_{a}}}{f}\right)=\operatorname{cov}\left(\theta, \frac{\hat{f_{a}}}{f}\right) . \quad \|
$$

Definition 4.2 (Talent sufficiency). The statistic $y$ is sufficient for $(y, z)$ for expected talent if $E(\theta \mid y, z, a)=E(\theta \mid y, a)$. 
Note that this is implied by the stronger condition that the density take the form

$$
h(y, z, \theta \mid a)=k(\theta \mid y, a) l(y, z \mid a)
$$

for some density $l$ and conditional density $k$.

Proposition 4.2. If $y$ is talent sufficient for $(y, z)$, then making $z$ available when $y$ already is does not alter marginal incentives.

Proof. Letting $\hat{f}(y \mid a)$ and $\hat{h}(y, z \mid a)$ denote the marginal densities of $y$ and $(y, z)$, respectively. The sufficiency condition,

$$
E(\theta \mid y, a)=E(\theta \mid y, z, a)
$$

implies that

$$
\begin{aligned}
E\left[E(\theta \mid y, z, a) \frac{\hat{h_{a}}(y, z \mid a)}{h(y, z \mid a)}\right] & =E\left[E(\theta \mid y, a) \frac{\hat{h_{a}}(y, z \mid a)}{h(y, z \mid a)}\right] \\
& =E\left[E(\theta \mid y, a) E\left[\frac{\hat{h}_{a}(y, z \mid a)}{h(y, z \mid a)} \mid y\right]\right]
\end{aligned}
$$

which together with the martingale condition,

$$
\frac{\hat{f_{a}}(y \mid a)}{f(y \mid a)}=E\left[\frac{\hat{h_{a}}(y, z \mid a)}{h(y, z \mid a)} \mid y\right],
$$

gives the result

$$
E\left[E(\theta \mid y, z, a) \frac{\hat{h_{a}}(y, z \mid a)}{h(y, z \mid a)}\right]=E\left[E(\theta \mid y, a) \frac{\hat{f_{a}}(y \mid a)}{f(y \mid a)}\right] .
$$

Evidently neither the conditions in Propositions 4.1 or 4.2 are necessary: both results are sufficient only. Proposition 4.1 implies that the sort of information irrelevant to incentives in a moral-hazard principal-agent context are also irrelevant in a career concerns context. However, since Holmstrom's sufficient statistic result is a necessary and sufficient one, Propositions 4.1 and 4.2 together imply that there are statistics irrelevant in the career concern model which would not be irrelevant in the principal-agent model.

\section{INCENTIVES AND INFORMATION ORDER}

\subsection{A lemma}

The impact of a Blackwell garbling on incentives is a priori less obvious. We first show by means of examples that a Blackwell garbling of the information system may raise welfare (unlike in the case of explicit incentives) and raise effort (unlike in the scalar additive-normal career concerns model). We then demonstrate that under some regularity conditions, a Blackwell garbling has an unambiguous (positive or negative) impact on incentives.

Example 5.1. Pass/Fail. Reporting whether a student passed or failed an exam rather than the grade amounts to garbling the information received by the "job market" (university committees, graduate schools, employers, ...). In circumstances in which the 
student is likely to get a grade near the pass/fail threshold, the pass/fail system induces more effort than a full disclosure of the grade.

This idea is easily illustrated by means of an example: Suppose that the student's grade is

$$
y=\theta+a,
$$

where $\theta \in\{0,1,5\}$. The three possible talents are equally likely. Effort is binary: $a \in\{0,1\}$. We assume that $c(1)-c(0) \in(1 / 3,2 / 3)$.

Under full disclosure of the grade $y$, by exerting the high effort the student increases her payoff only when $\theta=0$. Hence the maximum expected gain from working hard is $1 /$ 3 , and therefore the student shirks under full disclosure. Consider next a pass/fail system where only $z \in$ \{pass, fail\} is disclosed and in which the student obtains a pass if and only if $y \geqq 1$. The shirking outcome is no longer an equilibrium: The ex post payoff under shirking is then $\frac{2}{3}[(1+5) / 2]=2$ while that when working is equal to 3 . By contrast, $a=1$ is the (unique) equilibrium effort: The payoff to working is then equal to 2 (for sure), and the payoff to shirking is $4 / 3$. Pass/fail here raises the payoff of doing decently by pooling average performances with superior ones.

Example 5.2. Effort increasing wear and tear. In this example, the principal's payoff is the difference between the agent's unobservable marginal productivity (increasing in the agent's effort $a$ ) and the wear and tear of the machine the agent operates on. The agent's cost of effort is

$$
c(a)=(k-a)^{2},
$$

where $k>0$, so that the agent likes work up to a point. In the absence of observable variable, the agent picks effort $a=k$.

Now introduce a single observable statistic $z$ related to talent and effort - the ex post condition of the machinery, that is minus its wear and tear (the agent's marginal productivity is still unobserved). Let

$$
z=\theta-a+\varepsilon .
$$

be the ex post condition of the machinery. This is just a variant on the Holmstrom additive normal model, but where effort shifts the distribution of $z$ to the left instead of the right. Because of career concerns, effort is reduced below $k$ by the worker trying to pretend that the machines are in good shape because of talent rather than lack of use. Releasing information $z$ therefore reduces incentives.

Effort incentives are created by the agent trying to shift upwards the distribution of a random variable which will be interpreted by the market as good news for future productivity. Replacing a statistic $x$ by one with less information $T(x)$ will reduce effort if outside of $T$ but within $x$ there are statistics whose distribution the agent can shift upwards and which when greater will lead to an enhanced evaluation by the market about the agent's future productivity. More precisely, we have:

Lemma 5.1. If for a statistic $T=T(x), f_{a}(x \mid a) / f(x \mid a)$ and $\theta$ are conditionally positively correlated (respectively, negatively correlated) given $T$, marginal incentives are greater (respectively lower) when the market has information $T$ than when the market has information $x$. 
Proof. By the law of iterated expectations

$$
E\left[\theta \frac{\hat{f_{A}}(x \mid a)}{f(x \mid a)}\right]=E\left[E\left[\theta \frac{\hat{f_{A}}(x \mid a)}{f(x \mid a)} \mid T\right]\right] .
$$

Hence, by the assumed conditional correlation

$$
E\left[\theta \frac{\hat{f_{a}}(x \mid a)}{f(x \mid a)}\right] \underset{(\leqq)}{\geqq} E\left[E[\theta \mid T] \cdot E\left[\frac{\hat{f_{a}}(x \mid a)}{f(x \mid a)} \mid T\right]\right] .
$$

Denoting the marginal density of $T$ as $\hat{g}(T \mid a)$ and using the martingale condition (3.2), we have

$$
E\left[E[\theta \mid T] \cdot E\left[\frac{\hat{f_{a}}(x \mid a)}{f(x \mid a)} \mid T\right]\right]=E\left[E[\theta \mid T] \cdot \frac{\hat{g_{a}}(T \mid a)}{g(T \mid a)}\right]=E\left[\theta \frac{\hat{g_{a}}(T \mid a)}{g(T \mid a)}\right] . \quad \|
$$

We now provide two applications of this lemma to the cases in which (a) $x$ is a vector and $T(x)$ is a subvector obtained by deleting some components of $x$ (inclusive information systems), and (b) $x$ is a scalar.

\subsection{Inclusive information}

Suppose that there are two (possibly multivariate) observables $y$ and $z$. How do marginal incentives differ when the full statistic $x=(y, z)$ is reported and when only $T(x)=y$ is reported? It is intuitive that if a given statistic $y$ is already available, (i) the market's expectation of talent is increased when higher values of statistic $z$ are observed and (ii) if higher effort by the agent tends to increase that statistic (the MLRP property), then having that statistic in the market information set enhances effort. On the other hand, if the market's expectation of talent is increasing in a statistic that is decreased by effort, then having the statistic in the information set will reduce effort.

Consider the following conditions:

(a) $z$ is good news for $\theta$ conditional on $y\left(f(z, \theta \mid y, a)\right.$ is affiliated ${ }^{6}$ in $(z, \theta)$ for each $(y, a))$

(b) effort increases $z$ conditional on $y$ $(f(z \mid y, a)$ has MLRP in $(z, a)$ for each $y)$

$(\neg \mathrm{a}) z$ is bad news for $\theta$ conditional on $y$ (letting $\phi \equiv-\theta, f(z,-\phi \mid y, a)$ is affiliated in $(z, \phi)$ for each $(y, a))$

$(\neg$ b) effort decreases $z$ conditional on $y$ (letting $b \equiv-a, f(z \mid y,-b)$ has MLRP in $(z, b)$ for each $y)$.

Definition 5.1. We say news and effort are similarly ordered for $z$ conditional on $y$ if (a) and (b), or ( a) and ( b) hold, and oppositely ordered if (a) and ( $\neg$ b), or $(\neg$ a) and (b) hold.

Lemma 5.1 then implies:

6. In the case of a joint density on $\mathbb{R}^{l}$, two random variables are affiliated if $f\left(t \vee t^{\prime} \mid a\right) f\left(t \wedge t^{\prime} \mid a\right) \geqq f(t \mid a) f\left(t^{\prime} \mid a\right)$ where $t=(x, \theta), t \vee t^{\prime}$ is the component-wise maximum of $t$ and $t^{\prime}$ and $t \wedge t^{\prime}$ is the component-wise minimum. If $f$ is positive and $C^{2}$, then, from Topkis' theorem, affiliation is equivalent to $\partial^{2} \ln f / \partial t_{i} \partial t_{j} \geqq 0$ for all components $i \neq j$. 
Proposition 5.1 (Inclusive information). If effort and news are similarly ordered for $z$, conditionally on $y$, then the marginal incentive is greater when the market has information $(y, z)$ than $y$ alone. Similarly, if effort and news are oppositely ordered, then effort is greater when the market has information $y$ alone rather than $(y, z)$.

Example 5.3. Signal of talent. Start from an initial scalar signal $y$ given by

$$
y=\theta+a+\varepsilon
$$

and assume that $\varepsilon$ is a logconcave independent noise. The added signal $z$ about talent is independent from $a$ and $\varepsilon$ and the density $k(z, \theta)$ is affiliated. So, $z$ provides information about $\theta$ but not about $a$.

$$
\begin{aligned}
f(y, z, \theta \mid a) & =h(y-\theta-a) k(z, \theta), \\
\hat{f}(y, z \mid a) & =\int h(y-\theta-a) k(z, \theta) d \theta=m(y-a, z) .
\end{aligned}
$$

Evidently, given that $z$ and $\theta$ are affiliated, condition (a) holds. However, if $\varepsilon$ is logconcave and $(z, \theta)$ are affiliated, then $m(y-a, z)$ is affiliated, therefore condition $(\neg \mathrm{b})$ holds. It follows from Proposition 5.1 that marginal incentives are increased by reporting $y$ alone instead of $(y, z)$. Similarly, marginal incentives are increased by reporting $y$ alone if $z$ is bad news for $\theta$ (then ( a) and (b) hold).

Example 5.4. Increased precision. Again consider a scalar signal

$$
y=\theta+a+\varepsilon,
$$

and suppose that another statistic $z$ is informative about the noise $\varepsilon$. Specifically, let $\theta$ have density $k(\theta)$ independent and $(\varepsilon, z)$ have a joint density $h(\varepsilon, z)$. For simplicity assume $h$ is $\log$ concave in $\varepsilon$ for each $z$ and affiliated in $(\varepsilon, z)$. Then

$$
\begin{aligned}
f(y, z, \theta \mid a) & =h(y-\theta-a, z) k(\theta), \\
\hat{f}(y, z \mid a) & =\int h(y-\theta-a, z) k(\theta) d \theta .
\end{aligned}
$$

So, $(\neg \mathrm{a})$ holds, and $(\neg \mathrm{b})$ holds. It follows from Proposition 5.1 that marginal incentives are increased by reporting $(y, z)$ instead of $y$ alone. Note that marginal incentives would also increase if $z$ were bad news for $\varepsilon$ (in which case (a) and (b) would hold).

\subsection{Garbling a scalar performance}

Example 5.1 (pass/fail) is one in which incentives can be increased by coarsening a scalar information available to the market. We investigate therefore conditions under which incentives are not increased by any reduction of available information in the sense that

$$
g(z, \theta \mid a)=\int k(z \mid y) f(\theta, y \mid a) d y .
$$

This is read as follows: $y$ is available information; given a realization $y$, one adds noise by generating a random variable $z$ according to the conditional distribution $k(z \mid y)$. This definition means that there exists a joint distribution having the same marginal distributions as $(z, \theta)$ and $(y, \theta)$ and such that the probability distribution of $z$ conditional on $(y, \theta)$ is independent of $a$ and $\theta$. Although natural, the condition is stronger than we need. 
Note that (5.1) implies that there exists a joint distribution with the following properties: (i) the martingale condition holds and (ii) $E[\theta \mid y, z, a]$ independent of $z$. We know from Section 4 that $(y, z)$ and $y$ lead to the same marginal incentive. The question addressed here is how the marginal incentives compare under $y$ and under $z$ ?

Proposition 5.2. Assume that (i) $y$ (a scalan) and $\theta$ are affiliated $\left(\partial^{2} \ln f(\theta, y \mid a) /\right.$ $\partial \theta \partial y>0)$, (ii) $y$ is good news about effort $\left(f_{a}(y \mid a) / f(y \mid a)\right.$ is increasing in $\left.y\right)$, and (iii) (5.1) holds; then the marginal incentive is higher when the market has information $y$ than when it has information $z$. That is, incentives are higher under $y$ than under $z$.

Proof. Disclosing $y$ is equivalent to disclosing $\{y, z\}$ from (5.1). To apply Proposition 5.1 (disclosing $z$ is dominated by disclosing $(y, z)$ ), it suffices that $y$ is good news for $\theta$ given $z$ and $a$ increases $y$ given $z$. But

$$
f(\theta, y \mid z, a)=\frac{k(z \mid y) f(\theta, y \mid a)}{g(\theta, z \mid a)} \Rightarrow \frac{\partial^{2} \ln f(\theta, y \mid z, a)}{\partial \theta \partial y}=\frac{\partial^{2} \ln f(\theta, y \mid a)}{\partial \theta \partial y}>0,
$$

and so condition (a) holds. holds.

Also, $\hat{f}_{a}(y \mid z, a) / \hat{f}(y \mid z, a)=\hat{f}_{a}(y \mid a) / \hat{f}(y \mid a)$ is increasing in $y$ and so condition (b)

Example 5.5. Pass/Fail with regularity conditions on densities. Let

$$
x=\theta+a+\varepsilon,
$$

with $\theta$ and $\varepsilon$ having densities $g(\cdot)$ and $h(\cdot)$, and being independent and logconcave. Hence, $f(x, \theta \mid a)=h(x-\theta-a) g(\theta)$ which is affiliated in $(x, \theta)$ for each $a$ if $\varepsilon$ is logconcave. $x$ is the sum of two logconcave random variables and therefore also logconcave. Hence $\int f(x, \theta \mid a) d \theta=k(x-a)$ satisfies the monotone likelihood ratio condition. Therefore, under these conditions revealing grades is better than Pass/Fail. Note that without the logconcavity assumption it is not necessarily true that $E[\theta \mid x]$ is increasing.

\section{FISHER INFORMATION AND EXPONENTIAL FAMILIES}

Most applications of Holmstrom's (1982) model have assumed all random variables to be normally distributed. As a consequence the bound (2.1) holds with equality and explicit formulae become available. In this section we relax the normality assumption whilst retaining sufficient traces of it to be able to use the weaker concept of comparisons of Fisher information. We thereby observe that the Holmstrom approach is valid in broader contexts than the one it has been applied to.

\subsection{Normally distributed talent}

Consider first the additive scalar case $y=\theta+a+\varepsilon$ with $\theta \sim N\left(\bar{\theta}, \sigma_{\theta}^{2}\right)$. Then, regardless of the distribution of $\varepsilon$

$$
E[\theta \mid y, a]=\bar{\theta}+\sigma_{\theta}^{2} \frac{\hat{f_{a}}(y \mid a)}{f(y \mid a)} .
$$

As with the Cramer-Rao lower bound, in the normally distributed talent case, the bound (2.4) is therefore attained. 
Hence,

$$
\begin{aligned}
\operatorname{cov}\left(\theta, \frac{\hat{f_{a}}(y \mid a)}{f(y \mid a)}\right) & =\int E[\theta \mid y, a] \frac{\hat{f_{a}}(y \mid a)}{f(y \mid a)} \hat{f}(y \mid a) d y \\
& =\sigma_{\theta}^{2} \cdot \int\left(\frac{\hat{f_{a}}(y \mid a)}{f(y \mid a)}\right)^{2} \hat{f}(y \mid a) d y \\
& =\sigma_{\theta}^{2} \cdot E\left(\frac{\hat{f_{a}}(y \mid a)}{f(y \mid a)}\right)^{2} .
\end{aligned}
$$

So, in this case the marginal incentive is simply given by the product of the variance of $\theta$ and the Fisher information of the observed statistic. Note that since

$$
E\left(\frac{\hat{f_{a}(y \mid a)}}{f(y \mid a)}\right)^{2}=\left(\frac{1}{\sigma_{\theta}^{2}}\right)^{2} \cdot \operatorname{var}(E[\theta \mid y, a]),
$$

we can also write

$$
\operatorname{cov}\left(\theta, \frac{\hat{f_{a}}(y \mid a)}{f(y \mid a)}\right)=\frac{\operatorname{var}(E[\theta \mid y, a])}{\sigma_{\theta}^{2}}=\frac{\operatorname{var}(t)}{\sigma_{\theta}^{2}} .
$$

Therefore in equilibrium

$$
\operatorname{var}(t)=c^{\prime}\left(a^{*}\right) \cdot \sigma_{\theta}^{2}
$$

Remark. The above argument applies without modification when the agent's performance $(a+\theta)$ is assessed with noise by $n$ members of a jury

$$
y_{i}=\varphi_{i}\left(a+\theta, \varepsilon_{i}\right), \quad i=1, \ldots, m,
$$

as long as $\theta \sim N\left(\theta, \sigma_{\theta}^{2}\right)$. The noises $\varepsilon=\left(\varepsilon_{1}, \ldots, \varepsilon_{m}\right)$ can be arbitrary with $\varepsilon, \theta$ independently distributed.

\subsection{Exponentially distributed talent, multiplicative effort}

Let $y=\psi(a \theta, \varepsilon)$ with $\theta$ exponentially distributed with mean $\mu$. Let $x=a \theta$, the density of $x$ is given by $g(x \mid a)=(1 / a \mu) \exp [-(x / a \mu)]$. Hence, $g_{a} / g=(x-a \mu) / a^{2} \mu=(\theta-\mu) / a \mu$ and since $x$ is evidently sufficient for $y$, and using the martingale property, we have

$$
E[\theta \mid y, a]=a \mu \frac{\hat{f_{a}}(y \mid a)}{f(y \mid a)}+\mu .
$$

Hence,

$$
\operatorname{cov}\left(\theta, \frac{\hat{f_{a}}(y \mid a)}{f(y \mid a)}\right)=a \mu E\left(\frac{\hat{f_{a}}(y \mid a)}{f(y \mid a)}\right)^{2}
$$

Notice that nothing in the derivation of this formula rests on the nature of the random variable $\varepsilon$ or the function $\psi$. Any other Blackwell garbling of $x$ also leads to the formula. 
This means that for instance the incentive effects of information of the kind:

$$
z=\left\{\begin{array}{l}
\psi(a \theta, \varepsilon) \text { if some event (whose probability is independent of } a \text { ) occurs } \\
\zeta \text { if the event does not occur }
\end{array}\right.
$$

can easily be compared with the incentive effects of the information $y=\psi(a \theta, \varepsilon)$. [The interpretation is that the market does not know whether the agent has actually pursued the corresponding task. See our companion paper, Dewatripont et al. (1999), for a study of "fuzzy missions".] Since $z$ is clearly Blackwell less informative than $y$ and both are Blackwell less informative than $x$, we have

$$
\operatorname{cov}\left(\theta, \frac{\hat{h_{a}}(z \mid a)}{h(z \mid a)}\right)=a \mu E\left(\frac{\hat{h_{a}}(z \mid a)}{h(z \mid a)}\right)^{2},
$$

and since Fisher information is order preserving with respect to Blackwell information

$$
E\left(\frac{\hat{f_{a}}(y \mid a)}{f(y \mid a)}\right)^{2} \geqq E\left(\frac{\hat{h_{a}}(z \mid a)}{h(z \mid a)}\right)^{2}
$$

Hence,

$$
\operatorname{cov}\left(\theta, \frac{\hat{f_{a}}(y \mid a)}{f(y \mid a)}\right) \geqq \operatorname{cov}\left(\theta, \frac{\hat{h_{a}}(z \mid a)}{h(z \mid a)}\right)
$$

\subsection{Normally distributed noise}

We can also apply the same method as in Section 6.1 to the case where rather than bracketing effort and ability into a statistic with exponential family, one brackets ability and noise into a statistic with exponential family. The implication is that rather than view better information as being a more precise but manipulable signal, one views it as better prior non-manipulable information about ability. The distinction is exemplified by considering $y=a+\theta+\varepsilon$ with $\varepsilon$ normally distributed. We can bracket as $y=(a+\theta)+\varepsilon$ as above, or $y=\theta+(a+\varepsilon)$ as we do here. In this case, assuming $E \varepsilon=0$, and recalling ${ }^{7}$

$$
E[\theta \mid y, a]=y-a-\sigma_{\varepsilon}^{2} \frac{f_{\wedge}(y \mid a)}{f(y \mid a))} .
$$

We have

$$
\begin{aligned}
\operatorname{cov}\left(\theta, \frac{\hat{f_{a}}(y \mid a)}{f(y \mid a)}\right) & =\int E[E[\theta \mid y, a]] \frac{\hat{f_{a}}(y \mid a)}{f(y \mid a)} \hat{f}(y \mid a) d y \\
& =\int y f_{a}(y \mid a) d y-\sigma_{\varepsilon}^{2} \int\left(\frac{\hat{f_{a}}(y \mid a)}{f(y \mid a)}\right)^{2} \hat{f}(y \mid a) d y \\
& =1-\sigma_{\varepsilon}^{2} E\left(\frac{\hat{f_{a}}(y \mid a)}{f(y \mid a)}\right)^{2} .
\end{aligned}
$$

Again the comparison of Fisher informations suffices to compare marginal incentives.

7. This is a generalization of the "regression to the mode" result of Das and Mulder (1983). We are grateful to Andrew Chesher for this reference. In the additive case, assuming unimodality of $y$, it states that the expectation of the "second" observation of $y$ conditional on the "first" is greater than the first when the first lies below the mode. 


\section{SUMMARY AND CONCLUSION}

This paper has developed a career concerns model with multiple tasks and general signals. It has derived three main propositions on information systems. First, an agent's implicit incentives are not altered when only a sufficient statistic for the signal vector is disclosed. Like in the principal-agent model, the sufficient statistic property is a corollary of the fact that the agent's reward is determined (endogenously in the implicit incentives model, by design in the explicit incentives one) by the likelihood ratio. Second, given signal vectors $(y, z)$, disclosing $y$ only reduces (increases) marginal incentives if signals $z$ and efforts are similarly (oppositely) ordered. Similar (opposite) ordering obtains either when $z$ is good news for talent conditional on $y$ and efforts increase (decrease) $z$ conditional on $y$, or when $z$ is bad news about talent conditional on $y$ and efforts decrease (increase) $z$ conditional on $y$. Third, it may be possible to use the weaker concept of comparison of Fisher informations if one of the random variables has an exponential family distribution.

In our companion paper (Dewatripont et al. 1999), we apply the result that a Blackwell garbling reduces effort incentives when news and effort are similarly ordered to analyze several stylized facts about the functioning of Government agencies. We are able to identify precise conditions under which agencies perform better when they are assigned precise and clear missions, to paraphrase Wilson (1989)'s celebrated book on U.S. Government agencies. This paper also explains why agencies run by "professionals", with specific skills and external career concerns, may perform better and acquire more autonomy from their political principals.

Acknowledgements. The authors are grateful to Patrick Bolton, Juan Carrillo, Patrick Rey and a referee for helpful comments. Mathias Dewatripont acknowledges support from contract P4/28 of the Interuniversity Poles of Attraction Programme of the Belgian Federal State (Prime Minister's Office, Federal Office for Scientific, Technical and Cultural Affairs). Ian Jewitt acknowledges support from the ESRC.

\section{REFERENCES}

DAS, P. and MUlder, P. G. H. (1983), "Regression to the Mode", Statistica Neerlandica, 37, 15-21.

DEWATRIPONT, M., JEWITT, I. and TIROLE, J. (1997), "Missions and Accountability of Government Agencies" (Mimeo).

GROSSMAN, S. and HART, O. (1983), "An Analysis of the Principal-Agent Problem”, Econometrica, 51, $7-45$.

GUESNERIE, R. and LAFFONT, J. J. (1984), "A Complete Solution to a Class of Principal-Agent Problems with an Application to the Control of a Self-Managed Firm", Journal of Public Economics, 25, 329-369.

HOLMSTR@M, B. (1979), "Moral Hazard and Observability”, Bell Journal of Economics, 10, 74-91.

HOLMSTR $\odot$ M, B. (1982a), "Moral Hazard in Teams”, Bell Journal of Economics, 13, 324-340.

HOLMSTROM, B. (1982b), "Managerial Incentive Problems: A Dynamic Perspective", in Essays in Economics and Management in Honor of Lars Wahlbeck (Helsinki: Swedish School of Economics).

HOLMSTROM, B. and MILGROM, P. (1991), "Multi-Task Principal-Agent Analyses: Incentive Contracts, Asset Ownership, and Job Design", Journal of Law, Economics and Organization, 7, 24-52.

JEWITT, I. (1987), "Risk Aversion and the Choice between Risky Projects: The Preservation of Comparative Statics Results", Review of Economic Studies, 54, 73-86.

JEWITT, I. (1988), "Justifying the First-Order Approach to Principal-Agent Problems", Econometrica, 56, $1177-1190$.

JEWITT, I. (1997), "Information and Principal-Agent Problems" (Mimeo, University of Bristol).

KIM, S. K. (1995), "Efficiency of an Information System in an Agency Model”, Econometrica, 63, 89-102.

LEHMAN, E. (1988), “Comparing Location Experiments”, Annals of Statistics, 16, 521-533.

MIRRLEES, J. (1971), “An Exploration in the Theory of Optimum Income Taxation”, Review of Economic Studies, 38, 175-208.

MIRRLEES, J. (1999), "The Theory of Moral Hazard and Unobservable Behaviour, Part I", Review of Economic Studies, 66, 3-21

SHAVELL, S. (1979), "Risk Sharing and Incentives in the Principal and Agent Relationship", Bell Journal of Economics, 10, 55-73.

WILSON, J. Q. (1989), Bureaucracy: What Government Agencies Do and Why They Do It (New York: Basic Books). 